\title{
In-depth analysis of pneumococcal serotypes in Belgian children (2015-2018): Diversity, invasive disease potential, and antimicrobial susceptibility in carriage and disease
}

\author{
Stefanie Desmet ${ }^{\mathrm{a}, \mathrm{b}, 1, *}$, Ine Wouters ${ }^{\mathrm{c}, 1}$, Liesbet Van Heirstraeten ${ }^{\mathrm{d}}$, Philippe Beutels ${ }^{\mathrm{e}}$, Pierre Van Damme ${ }^{\mathrm{c}}$, \\ Surbhi Malhotra-Kumar ${ }^{\mathrm{d}}$, Piet Maes ${ }^{\mathrm{a}, \mathrm{b}}$, Jan Verhaegen ${ }^{\mathrm{a}, \mathrm{b}}$, Willy E Peetermans ${ }^{\mathrm{a}, \mathrm{b}}$, Katrien Lagrou ${ }^{\mathrm{a}, \mathrm{b}}$, \\ Heidi Theeten $^{c}$ \\ a Reference Centre for Pneumococci, University Hospitals Leuven, Herestraat 49, 3000 Leuven, Belgium \\ ${ }^{\mathrm{b}}$ Department of Microbiology, Immunology and Transplantation, KU Leuven, Herestraat 49, 3000 Leuven, Belgium \\ ${ }^{\mathrm{C}}$ Centre for the Evaluation of Vaccination, Vaccine and Infectious Disease Institute, University of Antwerp, Campus Drie Eiken, Universiteitsplein 1, 2610 Wilrijk, Belgium \\ ${ }^{\mathrm{d}}$ Laboratory of Medical Microbiology, Vaccine and Infectious Disease Institute, University of Antwerp, Campus Drie Eiken, Universiteitsplein 1, 2610 Wilrijk, Belgium \\ ${ }^{\mathrm{e}}$ Centre for Health Economics Research and Modelling Infectious Diseases, University of Antwerp, Campus Drie Eiken, Universiteitsplein 1, 2610 Wilrijk, Belgium
}

\section{A R T I C L E I N F O}

\section{Article history:}

Received 9 June 2020

Received in revised form 10 November 2020

Accepted 15 November 2020

Available online $\mathrm{xxxx}$

\begin{abstract}
A B S T R A C T
Background: Changes in serotype distribution have been described after the switch from the 13-valent pneumococcal conjugate vaccine (PCV13) to the 10 -valent pneumococcal conjugate vaccine (PCV10) in Belgium.

Aim: To describe serotype's invasive disease potential and the detailed evolution of serotype distribution and antimicrobial susceptibility of pneumococcal isolates (carriage and IPD) in children up to 30 months of age over a period during and after the vaccine switch (2015-2018).

Methods: S. pneumoniae strains isolated from the nasopharynx of healthy children attending day-care centres (DCCs) and strains from normally sterile sites of children with IPD were serotyped (Quellungreaction) and antimicrobial susceptibility testing was performed. Invasive disease potential was defined as the serotype-specific odds ratio (OR).

Results: The highly invasive (OR $>1$ ) serotypes $12 \mathrm{~F}, 1,3,24 \mathrm{~A} / \mathrm{B} / \mathrm{F}, 33 \mathrm{~F}, 19 \mathrm{~A}$, and $9 \mathrm{~N}$ were not frequently carried ( $<7.5 \%$ of carriage strains). Different serotypes dominated in carriage (23B, 23A, 11A, 15B) versus IPD $(12 \mathrm{~F}, 19 \mathrm{~A}, 10 \mathrm{~A}, 33 \mathrm{~F})$. PCV13 vaccine serotypes increased in carriage $(5.4 \%(25 / 463)$ in period 1 vs $10.3 \%(69 / 668)$ in period 3$)$ and in IPD (7.3\% (8/110 in period 1 vs $23.9 \%(34 / 142)$ in period 3$)$ due to an increase $(\mathrm{p}<0.01)$ in serotype $19 \mathrm{~A}$. The penicillin non-susceptibility of $19 \mathrm{~A}$ was lower $(\mathrm{p}=0.02)$ in carriage (6.8\%) than in IPD (23.5\%). Erythromycin and tetracycline non-susceptibility were more frequent $(\mathrm{p}<0.01)$ in IPD $(26.0 \% ; 23.0 \%)$ compared to carriage strains $(18.2 \% ; 14.5 \%)$ and penicillin nonsusceptibility increased over the three year study period (carriage: $13.4 \%, 19.8 \%, 18.5 \%, \mathrm{p}=0.05$; IPD: $11.8 \%, 15.0 \%, 20.4 \%, \mathrm{p}=0.02$ ).

Conclusion: Only some of the serotypes with high invasive disease potential (serotype $1,3,19 \mathrm{~A}$ ) in Belgium are included in PCV10 and/or PCV13. This reinforces the need for continuous monitoring, both in healthy children as in children with IPD, to better understand the dynamics of pneumococcal disease, to optimise the composition and implementation of PCVs.
\end{abstract}

(c) 2020 Elsevier Ltd. All rights reserved.

\section{Introduction}

S. pneumoniae often resides as a commensal in the human upper respiratory tract [1]. Nevertheless, asymptomatic carriage may

\footnotetext{
* Corresponding author.

1 Joint first author.
}

evolve to respiratory infections such as otitis media and pneumonia or invasive diseases such as bacteraemia and meningitis. These diseases are a serious health concern among children in which the highest carriage prevalence occurs [2]. In 2000, before pneumococcal conjugate vaccines (PCVs) were introduced, the global annual number of serious pneumococcal disease cases (pneumonia, meningitis, and bacteraemia) in children under five years of age was estimated to be 14.5 million [3]. In 2015, when PCVs had been 
implemented in 129 countries, this number had decreased to 9.2 million [4].

More than ninety-five pneumococcal serotypes exist and they vary in their capacity to colonise, invade, and activate the host immune system [5]. Furthermore, some serotypes are strongly associated with antimicrobial non-susceptibility [2]. Immunisation with PCVs provides direct protection against a number of clinically relevant serotypes [5]. In addition, indirect protection of unvaccinated individuals (herd effect) against pneumococcal disease is accomplished through reduced nasopharyngeal carriage and hence transmission of pneumococcal vaccine serotypes (VTs). However, several studies on carriage and invasive pneumococcal disease (IPD) reported on serotype replacement, i.e. VTs being largely replaced by non-VTs, which diminishes the magnitude of the vaccine effect [6-8].

Belgium initiated a free of charge universal paediatric immunisation PCV-programme according to a two plus one schedule (at 8 and 12 weeks, and 12 months of age) in 2007. The seven-valent vaccine (PCV7) was replaced by the thirteen valent (PCV13) in 2011, which was in turn replaced by the ten-valent vaccine (PCV10) in 2015-2016 [9]. The programme rapidly achieved high three dose coverage in children (2008-2009: >80\%; 2015-2016: $>94 \%$ ) [10-13].

Recent studies investigating paediatric IPD epidemiology and pneumococcal nasopharyngeal carriage in Belgium, reported an increase in PCV13 non-PCV10 serotypes shortly after the PCV13 to PCV10 vaccine switch [14-18].

The current study directly compares the evolution over time of pneumococcal serotype distribution and antimicrobial nonsusceptibility of pneumococci in healthy children and children with IPD, up to 30 months of age, between July 2015 and June 2018 , and focuses on serotype's invasive disease potential.

\section{Methods}

\subsection{Study design}

\subsubsection{Carriage monitoring}

The design of the carriage study was previously described in detail [15-17] and is summarised here. Healthy children between six and thirty months of age were recruited in day-care centres (DCCs) randomly selected in the three Belgian regions (Wallonia, Flanders, Brussels). Nasopharyngeal sampling was performed from March up to and including the first week of July in period 1 (2016) and from November up to the end of March in period 2 (20162017) and 3 (2017-2018). Trained nurses collected demographic and clinical characteristics and the vaccination status of the participating child. A single nasopharyngeal swab was taken with a flocked nylon fiber swab, transported in $1 \mathrm{ml}$ STGG (Skim milkTryptone-Glucose-Glycerol), and cultured or stored at $-80{ }^{\circ} \mathrm{C}$ within $24 \mathrm{~h}$. At the National Reference Centre (NRC) for pneumococci, nasopharyngeal samples were plated both directly and following BHI-enrichment on blood agar plates for detection of $S$. pneumoniae. If children carried different serotypes, all serotypes were taken into account.

\subsubsection{IPD surveillance}

All S. pneumoniae isolates sent to the NRC from July 2015 to June 2018 and collected from a normally sterile site (e.g. blood culture, cerebrospinal fluid, pleural fluid or synovial fluid) in children up to 30 months old were included in the study. Data collection was part of the passive surveillance network. From 2015 to 2018, the estimated representativeness of the surveillance for all Belgian IPD cases ranged between 89 and 92\% [18]. In total, 110 labs partici- pated in this period, which are located over the whole country. Results are analysed per epidemiological year (from July to June).

\subsection{Pneumococcal serotyping and antimicrobial susceptibility testing}

For all strains, S. pneumoniae identification was confirmed and serotyping performed by phase-contrast microscopy using the Quellung-reaction with serotype/serogroup-specific sera obtained from SSI Diagnostica (Hillerød, Denmark). Antimicrobial susceptibility testing was carried out by disk diffusion according to CLSI (2015-2017) [19] and EUCAST (2017-2018) [20] guidelines for oxacillin (penicillin), erythromycin, levofloxacin, and tetracycline. In case of positive oxacillin screen, minimal inhibitory concentration (MIC) was determined by Etest (BioMérieux, France) for penicillin. Penicillin MIC $>0.064 \mathrm{mg} / \mathrm{L}$ was interpreted as nonsusceptible.

\subsubsection{Statistics}

Chi-Square (Chi2) or Fisher's Exact Test (FET) were used in IBM SPSS Statistics 25 to compare variables between different groups, Spearman's Correlation Test was used to test the strength and direction of associations between variables; p-values less than 0.05 were considered to be statistically significant. To investigate the diversity of serotypes identified over the three year study period, the Simpson index of diversity and its 95\% confidence interval (95\%CI) was calculated via an online tool available at http:// www.comparingpartitions.info and using the formula:

\section{Simpson index of diversity $=1-\frac{1}{N(N-1)} \sum_{i=1}^{S} n_{i}\left(n_{i}-1\right)$ [36];}

with $\mathrm{N}=$ total number of isolates, $\mathrm{S}=$ total number of different serotypes, $n_{i}=$ number of isolates with serotype $i$. This index can be interpreted as the probability that two random pneumococcal isolates are different serotypes. Indices close to 1 indicating high diversity. Invasive disease potential was defined as the serotypespecific odds ratio (OR):

$O R=$ Number of IPD isolates of serotype

$x *$ Number of carriage isolates

that are not serotype $x$

Number of carriage isolates of serotype $x *$ Number of IPD isolates that are not serotype $x$ [22-24]; serotypes with an OR $>1$ were considered to have an increased probability to cause invasive disease. For carriage, in children attending DCCs, the following serotype clustering factor (SCF) was introduced: $S C F=\frac{\text { Number of pneumococcal strains with serotype } x}{\text { Number of DCCs where serotype } x \text { was identified }}$ in order to assess the distribution of serotypes over the different DCCs, and their tendency to cluster in particular DCCs. This factor was calculated per serotype, over the three year study period. SCF equals 1 for serotypes that were not found more than once per day-care centre in a season, or not more than once per season, and increases if a serotype is found in several children in the same day-care centre.

\section{Results}

\subsection{Study population}

Over the three successive periods, 1883 carriage isolates out of a total of 2817 samples from children attending DCCs and 365 isolates from children with IPD were included in the analyses. The gender and age distribution are shown in Table 1 (elaborate descriptives of the carriage study population were previously published [17], more details on both study populations can be found in Supplementary Table 1, 2). Regarding children attending DCCs, age distribution changed over the study period due to the inclusion of (on average) older children in Flanders in period 1 [15-17]. 
Table 1

Characteristics of children providing S. pneumoniae positive carriage samples and IPD samples by period, from 2016 till 2018 . (IPD: invasive pneumococcal disease).

\begin{tabular}{|c|c|c|c|c|c|c|c|c|c|c|c|c|c|}
\hline & & \multicolumn{6}{|c|}{ Carriage monitoring ${ }^{1}$} & \multicolumn{6}{|c|}{ IPD surveillance } \\
\hline & & \multicolumn{2}{|c|}{$\begin{array}{l}\text { Period 1: } \\
\text { spring } 2016 \\
(\mathrm{n}=463)\end{array}$} & \multicolumn{2}{|c|}{$\begin{array}{l}\text { Period 2: } \\
\text { winter } 2016- \\
2017(n=752)\end{array}$} & \multicolumn{2}{|c|}{$\begin{array}{l}\text { Period 3: } \\
\text { winter 2017- } \\
2018(n=668)\end{array}$} & \multicolumn{2}{|c|}{$\begin{array}{l}\text { Period 1: } \\
\text { July 2015- } \\
\text { June 2016 } \\
(\mathrm{n}=110)\end{array}$} & \multicolumn{2}{|c|}{$\begin{array}{l}\text { Period 2: } \\
\text { July 2016- } \\
\text { June } 2017 \\
(\mathrm{n}=113)\end{array}$} & \multicolumn{2}{|c|}{$\begin{array}{l}\text { Period 3: } \\
\text { July 2017- } \\
\text { June } 2018 \\
(\mathrm{n}=142)\end{array}$} \\
\hline & & $\mathrm{n}$ & $\%$ & $\mathrm{n}$ & $\%$ & $\mathrm{n}$ & $\%$ & $\mathrm{n}$ & $\%$ & $\mathrm{n}$ & $\%$ & $\mathrm{n}$ & $\%$ \\
\hline Number of centres ${ }^{2}$ & & 85 & - & 112 & - & 102 & - & 99 & - & 94 & - & 94 & - \\
\hline Gender & male & 236 & 51.0 & 373 & 49.6 & 310 & 46.4 & 59 & 53.6 & 61 & 54 & 82 & 58 \\
\hline \multirow[t]{4}{*}{ Age (months) } & $<6$ & 0 & 0 & 0 & 0 & 0 & 0 & 22 & 20.0 & 24 & 21.2 & 27 & 19.0 \\
\hline & $6-12$ & 56 & 12.1 & 183 & 24.3 & 151 & 22.6 & 45 & 40.9 & 39 & 34.5 & 58 & 40.8 \\
\hline & $13-24$ & 267 & 57.7 & 387 & 51.5 & 375 & 56.1 & 34 & 30.9 & 42 & 37.2 & 43 & 30.3 \\
\hline & $25-30$ & 140 & 30.2 & 182 & 24.2 & 142 & 21.3 & 9 & 8.2 & 8 & 7.1 & 14 & 9.9 \\
\hline
\end{tabular}

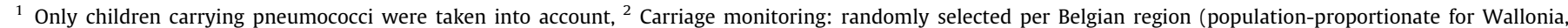
Flanders, Brussels), IPD surveillance: participating labs.

\subsection{Serotype distribution, overall study period}

Over the entire study period, 1883 carried strains representing 49 different serotypes and 365 IPD strains representing 46 different serotypes were detected. In both carriage and IPD a high proportion of serotypes not included in PCV13 (non-PCV13 serotypes) was found, respectively 93.6\% (1763/1883) and 83.0\% (303/365). $10.0 \%(188 / 1883)$ of carriage strains and $27.1 \%$ (99/365) of IPD strains were PCV15 serotypes (vaccine in development [25]; PCV13 serotypes plus serotype 22F and 33F). 36.0\% (678/1883) of carriage strains and 59.7\% (218/365) of IPD strains were PCV20 serotypes (vaccine in development [5]; PCV13 serotypes plus serotype $8,10 \mathrm{~A}, 11 \mathrm{~A}, 12 \mathrm{~F}, 15 \mathrm{~B}, 22 \mathrm{~F}, 33 \mathrm{~F}$ ).

Over the three year study period, the most frequently detected pneumococcal serotypes in carriage were 23B (15.6\%, 293/1883), $23 \mathrm{~A}(8.3 \%, 157 / 1883)$, and $11 \mathrm{~A}(7.8 \%, 146 / 1883)$ whereas in IPD serotype $12 \mathrm{~F}(15.9 \%, 58 / 365), 19 \mathrm{~A}(9.3 \%, 34 / 365)$, and $10 \mathrm{~A}(8.5 \%$, $31 / 365$ ) were most frequent. Serotypes $6 \mathrm{~A}, 7 \mathrm{C}, 11 \mathrm{~F}, 12 \mathrm{~A}, 15 \mathrm{~F}$,
$16 \mathrm{C}, 18 \mathrm{C}, 35 \mathrm{~A}, 38 \mathrm{~F}, 42$ were only detected in carriage and serotypes $5,8,9,11,15,16,19 \mathrm{~B}, 19 \mathrm{C}, 27,35$ exclusively in IPD. None of the IPD strains were serologically non-typeable, versus $2.0 \%(37 / 1883)$ of carriage strains.

In carriage, 30 serotypes had an SCF $>1$, of which the ten with the highest SCF (>1.5; range: 1.6-2.0) were 15A, 35B, 14, 31, 10A, 23A, 12F, 19A, 21, and 23B. Of these serotypes, 35B, 15A, 10A, 21, $23 \mathrm{~A}$, and $23 \mathrm{~B}$ were frequently carried serotypes (range: 5.0$15.6 \%$ of carriage isolates). According to Spearman's Correlation Test the SCF was associated with serotype carriage proportion (correlation coefficient $=0.81 ; \mathrm{p}<0.01$ ).

\subsection{Invasive disease potential}

In total 9 of the 36 serotypes detected both in carriage and IPD (12F, 1, 3, 24B, 24F, 24A, 33F, 19A, $9 \mathrm{~N}$ ) had an OR significantly higher than 1 (Fig. 1). The highest invasive disease potential was found for serotype $12 \mathrm{~F}(\mathrm{OR}=23.5,95 \% \mathrm{CI}=13.2-42.0$ ), one of the

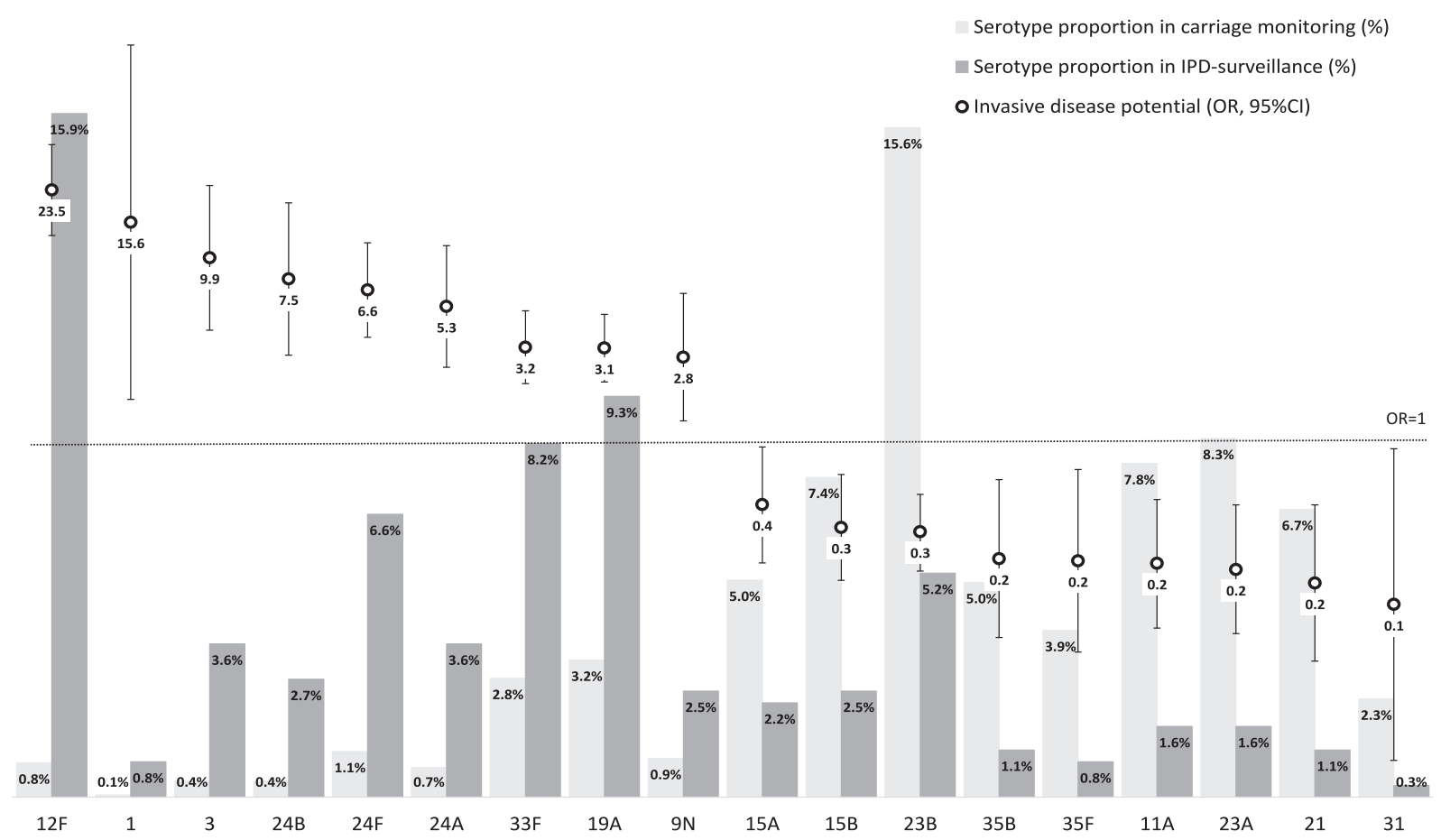

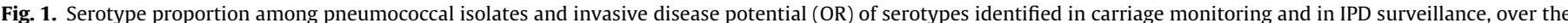

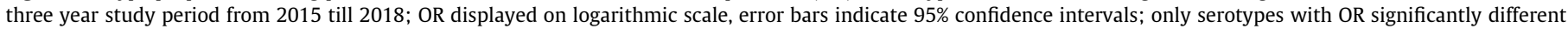
from 1 are shown, ORs of the other serotypes can be found in Supplementary Table 1 (OR and 95\%CI of serotype 10A: 1.5 (1.0-2.2)). 
dominating serotypes in IPD. For serogroup 24, the invasive disease potential was also high (range OR 5.3-7.5 for 24A, 24B, and 24F). The four serotypes most frequently identified in carriage $(11 \mathrm{~A}$, $15 \mathrm{~B}, 23 \mathrm{~A}, 23 \mathrm{~B}$ ) had an $\mathrm{OR}<1$, indicating low invasive disease potential. Among PCV13 vaccine serotypes, a high invasive disease potential was found for serotypes $1(\mathrm{OR}=15.6,95 \% \mathrm{CI}=1.6-150.4)$, $3(\mathrm{OR}=9.9,95 \% \mathrm{CI}=3.9-25.0)$, and $19 \mathrm{~A}(\mathrm{OR}=3.1,95 \% \mathrm{CI}=2.0-4.8)$, whereas the ORs of the remaining PCV13 vaccine serotypes, were not significantly different from 1 (Table 2) or could not be calculated.

\subsection{Serotype diversity and trends in time}

The evolutions in serotype distribution from period 1 to period 3 are summarized in Table 3. Simpson index of diversity showed higher levels of serotype diversity in each of the three periods for carriage strains than for IPD strains. No significant differences in serotype diversity were observed between carriage and IPD when comparing each of the three periods, except for period 1 in which the diversity of serotypes identified in IPD was lower than in carriage (see $95 \% \mathrm{CI}$ in Table 3 ).

At serotype level, serotype $6 \mathrm{C}$ was rare, but its carriage proportion significantly increased over the study period ( $\mathrm{p}<0.01$ ); from $0.9 \%(4 / 463)$ to $1.7 \%(13 / 752)$ and to $5.8 \%(39 / 668)$ and a similar but non-significant trend was seen among IPD (from 0.9\%; 1/110 in period 1 to $2.1 \% ; 3 / 142$ in period 3 ). Additionally, a decrease in the proportion of serotype $15 \mathrm{~A}$ was seen both in carriage and in IPD, which was only significant in carriage $(p=0.04)$; from $6.7 \%(31 / 463)$ to $5.5 \%(41 / 752)$ to $3.4 \%(23 / 668)$ in carriage and from $4.5 \%(5 / 110)$ to $0.9 \%(1 / 113)$ to $1.4 \%(2 / 142)$ in IPD.

However, the overall proportion of serotypes not included in PCV13 (Table 3) decreased significantly $(\mathrm{p}<0.01)$ in both carriage (from 94.6\% (438/463) to 89.7\% (599/688)) and IPD (from 92.7\% $(102 / 110)$ to $76.1 \%(108 / 142))$. The proportion of PCV10 vaccine serotypes, was already low at start and further decreased in carriage, from $4.5 \%(21 / 463)$ to $2.5 \%(17 / 668)$ ( $p<0.01$ ), whereas the proportion of PCV13-non-PCV10 vaccine types (3, 6A, 19A) significantly increased $(p<0.01)$ both in carriage and IPD due to a significant increase $(\mathrm{p}<0.01)$ in serotype $19 \mathrm{~A}$ over the study period; from $0.4 \%(2 / 463)$ to $1.5 \%(11 / 752)$ to $7.0 \%(47 / 668)$ in carriage and from $2.7 \%(3 / 110)$ to $5.3 \%(6 / 113)$ to $17.6 \%(25 / 142)$ in IPD. Apart from $6 C, 15 \mathrm{~A}$ and $19 \mathrm{~A}$, no other serotype proportions significantly changed over the study period.

\subsection{Antimicrobial non-susceptibility}

Non-susceptibility of pneumococcal strains against levofloxacin was inexistent in both carriage and in IPD (Table 4). Over the three

Table 2

Invasive disease potential (OR) of serotypes identified in carriage monitoring and in IPD surveillance, over the three year study period from 2015 till 2018.

\begin{tabular}{|c|c|c|c|c|c|c|c|c|}
\hline \multirow[t]{2}{*}{ Serotype } & & \multicolumn{2}{|c|}{$\begin{array}{l}\text { Carriage monitoring } \\
(\mathrm{N}=1883)\end{array}$} & \multicolumn{2}{|c|}{$\begin{array}{l}\text { IPD surveillance } \\
(\mathrm{N}=365)\end{array}$} & \multirow[t]{2}{*}{ OR } & \multicolumn{2}{|l|}{$95 \% \mathrm{CI}$} \\
\hline & & $\mathrm{n}$ & $\%$ & $\mathrm{n}$ & $\%$ & & Lower bound & Upper bound \\
\hline $12 \mathrm{~F}$ & PCV20 VT & 15 & 0.8 & 58 & 15.9 & 23.5 & 13.2 & 42.0 \\
\hline 1 & PCV10 VT & 1 & 0.1 & 3 & 0.8 & 15.6 & 1.6 & 150.4 \\
\hline 3 & PCV13 VT & 7 & 0.4 & 13 & 3.6 & 9.9 & 3.9 & 25.0 \\
\hline $24 \mathrm{~B}$ & NVT & 7 & 0.4 & 10 & 2.7 & 7.5 & 2.9 & 20.0 \\
\hline $24 \mathrm{~F}$ & NVT & 20 & 1.1 & 24 & 6.6 & 6.6 & 3.6 & 12.0 \\
\hline $24 \mathrm{~A}$ & NVT & 13 & 0.7 & 13 & 3.6 & 5.3 & 2.4 & 11.6 \\
\hline $12 \mathrm{~A}$ & NVT & 1 & 0.1 & 1 & 0.3 & 5.2 & 0.3 & 82.9 \\
\hline $7 \mathrm{~B}$ & NVT & 1 & 0.1 & 1 & 0.3 & 5.2 & 0.3 & 82.9 \\
\hline $12 \mathrm{~B}$ & NVT & 3 & 0.2 & 2 & 0.5 & 3.5 & 0.6 & 20.7 \\
\hline $23 \mathrm{~F}$ & PCV7 VT & 3 & 0.2 & 2 & 0.5 & 3.5 & 0.6 & 20.7 \\
\hline $33 \mathrm{~F}$ & PCV15 VT & 52 & 2.8 & 30 & 8.2 & 3.2 & 2.0 & 5.0 \\
\hline $19 A$ & PCV13 VT & 60 & 3.2 & 34 & 9.3 & 3.1 & 2.0 & 4.8 \\
\hline 14 & PCV7 VT & 5 & 0.3 & 3 & 0.8 & 3.1 & 0.7 & 13.1 \\
\hline $9 \mathrm{~N}$ & NVT & 17 & 0.9 & 9 & 2.5 & 2.8 & 1.2 & 6.3 \\
\hline 29 & NVT & 4 & 0.2 & 2 & 0.5 & 2.6 & 0.5 & 14.2 \\
\hline 9L & NVT & 2 & 0.1 & 1 & 0.3 & 2.6 & 0.2 & 28.6 \\
\hline $22 \mathrm{~F}$ & PCV15 VT & 16 & 0.8 & 7 & 1.9 & 2.3 & 0.9 & 5.6 \\
\hline 38 & NVT & 23 & 1.2 & 9 & 2.5 & 2.0 & 0.9 & 4.5 \\
\hline $33 \mathrm{~A}$ & NVT & 6 & 0.3 & 2 & 0.5 & 1.7 & 0.4 & 8.6 \\
\hline $10 \mathrm{~A}$ & PCV20 VT & 111 & 5.9 & 31 & 8.5 & 1.5 & 1.0 & 2.2 \\
\hline $17 \mathrm{~F}$ & NVT & 11 & 0.6 & 2 & 0.5 & 0.9 & 0.2 & 4.3 \\
\hline $19 \mathrm{~F}$ & PCV7 VT & 42 & 2.2 & 6 & 1.6 & 0.7 & 0.3 & 1.7 \\
\hline $15 C$ & PCV20 VT & 78 & 4.1 & 10 & 2.7 & 0.7 & 0.3 & 1.3 \\
\hline 34 & NVT & 8 & 0.4 & 1 & 0.3 & 0.6 & 0.1 & 5.2 \\
\hline $16 \mathrm{~F}$ & NVT & 64 & 3.4 & 6 & 1.6 & 0.5 & 0.2 & 1.1 \\
\hline $6 C$ & NVT & 56 & 3.0 & 5 & 1.4 & 0.5 & 0.2 & 1.1 \\
\hline $15 \mathrm{~A}$ & NVT & 95 & 5.0 & 8 & 2.2 & 0.4 & 0.2 & 0.9 \\
\hline $10 \mathrm{~B}$ & NVT & 30 & 1.6 & 2 & 0.5 & 0.3 & 0.1 & 1.4 \\
\hline $15 B$ & PCV20 VT & 140 & 7.4 & 9 & 2.5 & 0.3 & 0.2 & 0.6 \\
\hline $23 B$ & NVT & 293 & 15.6 & 19 & 5.2 & 0.3 & 0.2 & 0.5 \\
\hline $35 B$ & NVT & 94 & 5.0 & 4 & 1.1 & 0.2 & 0.1 & 0.6 \\
\hline $35 \mathrm{~F}$ & NVT & 73 & 3.9 & 3 & 0.8 & 0.2 & 0.1 & 0.7 \\
\hline $11 \mathrm{~A}$ & PCV20 VT & 146 & 7.8 & 6 & 1.6 & 0.2 & 0.1 & 0.5 \\
\hline $23 \mathrm{~A}$ & NVT & 157 & 8.3 & 6 & 1.6 & 0.2 & 0.1 & 0.4 \\
\hline 21 & NVT & 126 & 6.7 & 4 & 1.1 & 0.2 & 0.1 & 0.4 \\
\hline 31 & NVT & 43 & 2.3 & 1 & 0.3 & 0.1 & 0.0 & 0.9 \\
\hline
\end{tabular}

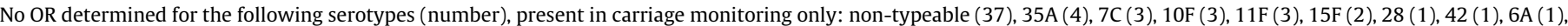

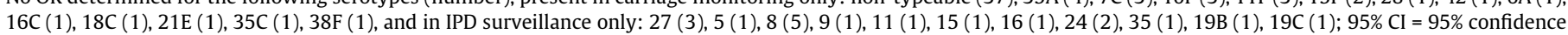

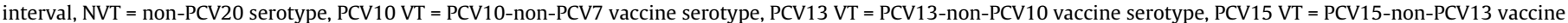

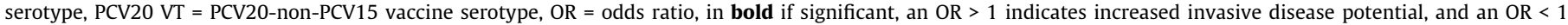
indicates decreased invasive disease potential 
Table 3

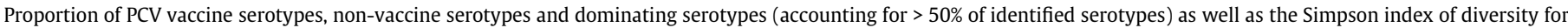

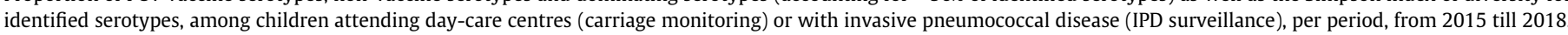

\begin{tabular}{|c|c|c|c|c|c|c|}
\hline & \multicolumn{3}{|l|}{ Carriage monitoring } & \multicolumn{3}{|l|}{ IPD surveillance } \\
\hline & Serotype & $\mathrm{n}$ & $\%$ & Serotype & $\mathrm{n}$ & $\%$ \\
\hline \multirow[t]{13}{*}{ Period 1: 2015-2016 ${ }^{1}$} & all serotypes & 463 & 100.0 & all serotypes & 110 & 100.0 \\
\hline & PCV10 vaccine serotypes & 21 & 4.5 & PCV10 vaccine serotypes & 3 & 2.7 \\
\hline & PCV13 vaccine serotypes & 25 & 5.4 & PCV13 vaccine serotypes & 8 & 7.3 \\
\hline & PCV13-non-PCV10 serotypes & 4 & 0.9 & PCV13-non-PCV10 serotypes & 5 & 4.5 \\
\hline & Non-PCV13 serotypes & 438 & 94.6 & Non-PCV13 serotypes & 102 & 92.7 \\
\hline & Dominating serotypes & & & Dominating serotypes & & \\
\hline & 23B & 64 & 13.8 & $12 \mathrm{~F}$ & 20 & 18.2 \\
\hline & $23 \mathrm{~A}$ & 49 & 10.6 & $10 \mathrm{~A}$ & 13 & 11.8 \\
\hline & $11 \mathrm{~A}$ & 40 & 8.6 & $33 \mathrm{~F}$ & 12 & 10.9 \\
\hline & $15 B$ & 33 & 7.1 & $15 B$ & 6 & 5.5 \\
\hline & $15 \mathrm{~A}$ & 31 & 6.7 & $24 \mathrm{~F}$ & 5 & 4.5 \\
\hline & $10 \mathrm{~A}$ & 27 & 5.8 & $15 \mathrm{~A}$ & 5 & 4.5 \\
\hline & $\begin{array}{l}\text { Simpson's diversity index } \\
(95 \% \mathrm{CI})\end{array}$ & $\begin{array}{r}0.92 \\
(0.85\end{array}$ & & $\begin{array}{l}\text { Simpson's diversity index } \\
(95 \% \mathrm{CI})\end{array}$ & $\begin{array}{l}0.78 \\
(0.70-0.86)\end{array}$ & \\
\hline \multirow[t]{13}{*}{ Period 2: 2016-2017 } & all serotypes & 752 & 100.0 & all serotypes & 113 & 100.0 \\
\hline & PCV10 vaccine serotypes & 14 & 1.9 & PCV10 vaccine serotypes & 9 & 8.0 \\
\hline & PCV13 vaccine serotypes & 26 & 3.5 & PCV13 vaccine serotypes & 20 & 17.7 \\
\hline & PCV13-non-PCV10 serotypes & 12 & 1.6 & PCV13-non-PCV10 serotypes & 11 & 9.7 \\
\hline & Non-PCV13 serotypes & 726 & 96.5 & Non-PCV13 serotypes & 93 & 82.3 \\
\hline & Dominating serotypes & & & Dominating serotypes & & \\
\hline & 23B & 133 & 17.7 & $12 \mathrm{~F}$ & 17 & 15.0 \\
\hline & 15B & 62 & 8.3 & $10 \mathrm{~A}$ & 11 & 9.7 \\
\hline & $10 \mathrm{~A}$ & 59 & 7.9 & $33 \mathrm{~F}$ & 10 & 8.8 \\
\hline & $23 \mathrm{~A}$ & 58 & 7.7 & $23 \mathrm{~B}$ & 6 & 5.3 \\
\hline & 21 & 54 & 7.2 & $19 A$ & 6 & 5.3 \\
\hline & $11 \mathrm{~A}$ & 51 & 6.8 & $24 \mathrm{~B}$ & 6 & 5.3 \\
\hline & $\begin{array}{l}\text { Simpson's diversity index } \\
(95 \% \mathrm{CI})\end{array}$ & $\begin{array}{c}0.91 \\
(0.85\end{array}$ & & $\begin{array}{l}\text { Simpson's diversity index } \\
(95 \% \mathrm{CI})\end{array}$ & $\begin{array}{l}0.80 \\
(0.72-0.87)\end{array}$ & \\
\hline \multirow[t]{13}{*}{ Period 3: 2017-2018 } & all serotypes & 668 & 100.0 & all serotypes & 142 & 100.0 \\
\hline & PCV10 vaccine serotypes & 17 & 2.5 & PCV10 vaccine serotypes & 3 & 2.1 \\
\hline & PCV13 vaccine serotypes & 69 & 10.3 & PCV13 vaccine serotypes & 34 & 23.9 \\
\hline & PCV13-non-PCV10 serotypes & 52 & 7.8 & PCV13-non-PCV10 serotypes & 31 & 21.8 \\
\hline & Non-PCV13 serotypes & 599 & 89.7 & Non-PCV13 serotypes & 108 & 76.1 \\
\hline & Dominating serotypes & & & Dominating serotypes & & \\
\hline & $23 \mathrm{~B}$ & 96 & 14.4 & $19 \mathrm{~A}$ & 25 & 17.6 \\
\hline & $11 \mathrm{~A}$ & 55 & 8.2 & $12 \mathrm{~F}$ & 21 & 14.8 \\
\hline & $23 \mathrm{~A}$ & 50 & 7.5 & $24 \mathrm{~F}$ & 15 & 10.6 \\
\hline & 21 & 49 & 7.3 & $23 B$ & 9 & 6.3 \\
\hline & $19 \mathrm{~A}$ & 47 & 7.0 & $33 \mathrm{~F}$ & 8 & 5.6 \\
\hline & $15 \mathrm{~B}$ & 45 & 6.7 & $10 \mathrm{~A}$ & 7 & 4.9 \\
\hline & $\begin{array}{l}\text { Simpson's diversity index } \\
(95 \% \mathrm{CI})\end{array}$ & $\begin{array}{r}0.90 \\
(0.8\end{array}$ & & $\begin{array}{l}\text { Simpson's diversity index } \\
(95 \% \mathrm{CI})\end{array}$ & $\begin{array}{l}0.81 \\
(0.74-0.88)\end{array}$ & \\
\hline
\end{tabular}

PCV10 vaccine serotypes include serotypes: 4, 6B, 9V, 14, 18C, 19F, 23G, 1, 5, 7F, PCV13 vaccine serotypes include PCV10 vaccine serotypes plus: 3, 6A, 19A, 95\% CI = 95\% confidence interval.

${ }^{1}$ Period 1 represents spring 2016 in carriage and 1 July 2015 - 1 June 2016 in IPD.

Table 4

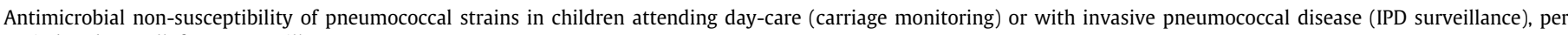
period and overall, from 2015 till 2018.

\begin{tabular}{|c|c|c|c|c|c|c|c|c|c|c|c|}
\hline \multirow[b]{2}{*}{$\begin{array}{l}\text { Non- } \\
\text { susceptibility } \\
\text { against }\end{array}$} & & \multicolumn{5}{|c|}{ Carriage monitoring } & \multicolumn{5}{|c|}{ IPD surveillance } \\
\hline & & $\begin{array}{l}\text { Period 1: } \\
2016 \\
(N=463)\end{array}$ & $\begin{array}{l}\text { Period 2: } \\
2016-2017 \\
(\mathrm{~N}=752)^{1}\end{array}$ & $\begin{array}{l}\text { Period 3: } \\
2017-2018 \\
(\mathrm{~N}=668)^{2}\end{array}$ & $\begin{array}{l}\text { Overall } \\
(\mathrm{N}=1883)\end{array}$ & $\begin{array}{l}\text { p-value } \\
\text { Chi }^{2} \text { for } \\
\text { trend }\end{array}$ & $\begin{array}{l}\text { Period 1: } \\
2015-2016 \\
(\mathrm{~N}=110)\end{array}$ & $\begin{array}{l}\text { Period 2: } \\
2016-2017 \\
(\mathrm{~N}=113)\end{array}$ & $\begin{array}{l}\text { Period 3: } \\
2017-2018 \\
(N=142)\end{array}$ & $\begin{array}{l}\text { Overall } \\
(\mathrm{N}=365)\end{array}$ & $\begin{array}{l}\mathrm{p} \text {-value } \\
\mathrm{Chi}^{2} \text { for } \\
\text { trend }\end{array}$ \\
\hline \multirow[t]{2}{*}{ Erythromycin } & $\mathrm{n}$ & 80 & 116 & 146 & 342 & 0.02 & 27 & 30 & 38 & 95 & 0.23 \\
\hline & $\%$ & 17.3 & 15.5 & 22.0 & 18.2 & & 24.5 & 26.5 & 26.8 & 26.0 & \\
\hline \multirow[t]{2}{*}{ Tetracycline } & $\mathrm{n}$ & 54 & 86 & 133 & 273 & $<0.01$ & 21 & 23 & 40 & 84 & 0.01 \\
\hline & $\%$ & 11.7 & 11.5 & 20.0 & 14.5 & & 19.1 & 20.4 & 28.2 & 23.0 & \\
\hline \multirow[t]{2}{*}{ Penicillin } & $\mathrm{n}$ & 62 & 148 & 123 & 333 & 0.05 & 13 & 17 & 29 & 59 & 0.02 \\
\hline & $\%$ & 13.4 & 19.8 & 18.5 & 17.7 & & 11.8 & 15.0 & 20.4 & 16.2 & \\
\hline
\end{tabular}

Non-susceptibility against levofloxacin was inexistent.

$\mathrm{nS}=$ non-susceptible; $\mathrm{S}=$ susceptible.

1 Non-susceptibility against erythromycin, tetracycline and penicillin was determined for 747,749 and 746 Sp-isolates respectively, ${ }^{2}$ Non-susceptibility against erythromycin, tetracycline, and penicillin was determined for 664, 666 and 666 Sp-isolates respectively.

year study period, non-susceptibility against erythromycin and tetracycline was significantly more frequent $(\mathrm{p}<0.01)$ in IPD (26.0\% (95/365) for erythromycin and 23.0\% (84/365) for tetracy- cline) than in carriage (18.2\% (342/1883) for erythromycin and $14.5 \%(273 / 1883)$ for tetracycline). Penicillin non-susceptibility was similar in IPD strains $(16.2 \% ; 59 / 365)$ and carriage strains 
(17.7\%; 333/1883). The five pneumococcal serotypes that were most frequently non-susceptible to penicillin were also similar in carriage and IPD; 11A, 23B, 24A, 24B, and 24F. Remarkably, the non-susceptibility of serotype $19 \mathrm{~A}$ against penicillin was three times higher $(\mathrm{p}=0.02)$ in IPD $(23.5 \%, 8 / 34)$ than in carriage $(6.8 \%, 4 / 59)$. Analyzing trends over time (Table 4) showed that non-susceptibility for penicillin and tetracycline significantly increased over the study period in carriage and IPD. Erythromycin non-susceptibility also increased, but the increase was only significant in carriage.

\section{Discussion}

During and immediately after the PCV13-to-PCV10 vaccination programme switch (2015-2018), we report different predominant serotypes in IPD and carriage in healthy children. However similar trends in serotype distribution and antimicrobial nonsusceptibility are noticed in IPD and carriage over this three years period. For a majority of the serotypes no significant change in serotype proportion was detected, except for serotype 19A, 15A and $6 \mathrm{C}$. Interestingly, some of the serotypes with high invasive disease potential (e.g. serotype $12 \mathrm{~F}$ and $33 \mathrm{~F}$ ) are not included in the current available PCVs, but will be included in one or both of the new upcoming PCVs (PCV15/PCV20). The information about serotype distribution in carriage and IPD is important to support the policy makers in decisions about the most appropriate pneumococcal vaccination programme, and can direct the development of new vaccines.

The sizes of both studied child population samples are large (DCC; $\mathrm{N}=2817$ and IPD; $\mathrm{N}=365$ ) and representative. Day-care attendance is common in Belgian children (in 2017: $52.6 \%$ of $0-$ 30 months old children in Flanders [26]; $36.1 \%$ of $0-36$ months old children in Wallonia [27]; $29.9 \%$ of $0-36$ months old children in Brussels [27]) and the participating DCCs were randomly selected over the three Belgian regions (Wallonia, Flanders, Brussels). Therefore, the DCC-based sample can be considered representative of healthy children aged 6-30 months residing in daycare. The IPD surveillance is with its high and constant estimated laboratory coverage (range: $89-92 \%$ ) also representative of the Belgian IPD cases over the three year study period.

Calculations on invasive disease potential are interesting in the context of pneumococcal vaccine composition and implementation of a vaccination programme. Serotypes differ in their probability to cause IPD. Hence, the inclusion of more invasive serotypes in a vaccine will determine the extent to which it can prevent IPD. As expected and in accordance with Dutch and Swedish study reports [22,24], our data (Fig. 1, Table 2) clearly indicated a higher invasive disease potential $(\mathrm{OR}>1)$ for serotypes dominating in IPD $(12 \mathrm{~F}$, $19 \mathrm{~A}, 33 \mathrm{~F}, 24 \mathrm{~F})$ and a lower invasive disease potential $(\mathrm{OR}<1)$ for serotypes dominating in carriage (23B, 23A, 11A, 15B).

Serotype $12 \mathrm{~F}$ contributed most to IPD over the three year study period, except for period 3 in which serotype 19A became the most frequent IPD-type. Serotype $12 \mathrm{~F}$ was also rare in carriage (15/1883; $0.8 \%)$, and therefore had the highest invasive disease potential $(\mathrm{OR}=23.5,95 \% \mathrm{CI}=13.2-42.0)$. It is important to know that the upcoming higher-valent PCV20 will target serotype $12 \mathrm{~F}$ and some other serotypes dominating IPD in Belgium (10A, 11A, 15B, 33F, but only 33F with OR > 1)) [28]. The PCV20-non-PCV13 serotypes are responsible for $38.6 \%$ and $24.8 \%$ of strains in respectively IPD and carriage in Belgian children, whereas PCV15-non-PCV13 (22F and $33 \mathrm{~F}$ ) serotypes represent $8.8 \%$ of IPD and $2.9 \%$ of carriage. This emphasizes the importance of dual surveillance of serotypes, both in terms of carriage and IPD.

Among PCV13 vaccine types, OR could only be estimated for serotypes 1, 3, and 19A. All three had a significant $\mathrm{OR}>1$ and are thus considered to present a high risk of causing IPD. The OR we calculated for serotypes $1(\mathrm{OR}=15.6,95 \% \mathrm{CI}=1.6-150.4)$ and $3(\mathrm{OR}=9.9,95 \% \mathrm{CI}=3.9-25.0)$ were within the range described by other reports (range OR for serotype 1: 8.8-33.4; range OR for serotype 3: $0.9-18.8$ ), but for $19 \mathrm{~A}$, the $\mathrm{OR}$ we calculated $(\mathrm{OR}=3.1,95 \% \mathrm{CI}=2.0-4.8)$ was higher than reported elsewhere (range OR: $1.0-2.4$ ) $[22,23,24,29]$. Similar to a Swedish report, we found a borderline non-significant $\mathrm{OR}$ for serotype $10 \mathrm{~A}$ $(\mathrm{OR}=1.5,95 \% \mathrm{CI}=1.0-2.2)$, whereas a Dutch study reported a significant OR of $1.7(95 \% \mathrm{CI}=1.1-2.7)$ for this serotype $[22,24]$.

Similar serotypes with high invasive disease potential were reported in a meta-analysis of 13 carriage/IPD settings in Europe, North-America, Latin-America, and Africa, which adopted serotype $19 \mathrm{~A}$ as the reference type for invasive disease potential [30]. Their results among children up to twenty-three months of age showed that, serotypes $7 \mathrm{~F}, 12 \mathrm{~F}, 1$, and 3 had a higher invasive disease potential than 19A, corroborating our findings except that serotype 7F was absent in our study population. Other PCV13 vaccine serotypes with a significant $O R$ identified in the meta-analysis were $6 \mathrm{~A}$, $23 \mathrm{~F}, 6 \mathrm{~B}$, and $19 \mathrm{~F}$, with an $\mathrm{OR}<1$. In our study only $19 \mathrm{~F}$ had an OR estimate lower than 19A. This difference may be due to the different period of the studies post PCV-introduction, or the use of a different PCV. In Belgium, after PCV13-introduction in 2011, serotypes $6 \mathrm{~A}, 6 \mathrm{~B}, 23 \mathrm{~F}$, and $19 \mathrm{~F}$ almost completely disappeared as paediatric IPD causing serotypes, and only $19 \mathrm{~F}$ is still carried at low level in healthy children.

Even though it was expected that a PCV13-to-PCV10 vaccination programme switch would have a more immediate impact on serotype distribution in childhood carriage than IPD, the current study suggests a simultaneous change. Indeed, the proportion of 19A significantly increased in parallel in carriage and in IPD from 2015 to 2016 onward (Table 3). This increase was accompanied by a simultaneous increase of the non-vaccine serotype $6 \mathrm{C}$ proportion, though at much lower level, suggesting absence of PCV10induced cross-protection against serotype 6C. Additionally, a simultaneous decrease of the non-PCV13 serotype 15A was seen, also only significant in carriage. Any hypothesis explaining this coincidence remains to be found, and it needs to be confirmed in future follow-up. Also unexplained, the proportion of PCV10 vaccine serotypes significantly decreased over the study period in carriage but not in IPD. However, it is difficult to make conclusions on evolution of PCV10 serotypes over the study period, as numbers in carriage and IPD were already low at the beginning of the study.

In a Dutch study investigating pneumococcal carriage in PCV10vaccinated 24-month-old children and in children with IPD who were up to five years old [24], serotypes 6C, 23B, 11A, 15B, and $23 \mathrm{~A}$ were the most frequently reported serotypes in carriage. These are with exception of $6 \mathrm{C}$, the same frequent serotypes as in our study. The most frequently identified IPD serotypes reported in this Dutch study were serotypes $19 \mathrm{~A}, 3,8,6 \mathrm{C}, 27$, and 33F, whereas in our study the dominating serotypes in 2017-2018 were $19 \mathrm{~A}, 12 \mathrm{~F}$, $24 \mathrm{~F}, 23 \mathrm{~B}, 33 \mathrm{~F}$, and $10 \mathrm{~A}$.

In addition, contrasting our study results, the Dutch study reported a decline in carriage of serotype 19A as soon as PCV10 was implemented, however, it remained one of the serotypes contributing most to IPD (besides serotypes 3 and 8). Whether the increase of serotype 19A in Belgian IPD and carriage resulted from the serotypes' natural evolution or from a causal relationship with vaccine switch remains to be further investigated. As from mid2019, decision was made to switch back from PCV10 to PCV13 in Belgium, further follow up of IPD and carriage serotype distribution will be very important.

The penicillin non-susceptibility in serotype 19A IPD strains $(23.5 \%, 8 / 34)$ was lower than in the pre-PCV13 period (in 2011: $38.6 \%$ of Belgian serotype 19A IPD strains in children were nonsusceptible, data NRC). Interestingly, penicillin non-susceptibility 
was less frequent among serotype 19A carriage strains (6.8\%, 4/59) than in 19A IPD-strains which may suggest that other serotype $19 \mathrm{~A}$ clones are involved in IPD than in carriage. By means of wholegenome sequencing of serotype 19A strains of both carriage and IPD, we will further investigate this hypothesis. The microepidemiology of these $19 \mathrm{~A}$ strains will be important to better understand the recent evolutions. For serogroup 24 (also highly invasive), penicillin non-susceptibility was more frequent than for serotype $19 \mathrm{~A}$, ranging from $53.8 \%(7 / 13,24 \mathrm{~A})$ to $70.0 \%(14 / 20$, $24 \mathrm{~F})$ in carriage and from $40.0 \%(4 / 10,24 \mathrm{~B})$ to $76.9 \%(10 / 13,24 \mathrm{~A})$ in IPD. In France, shortly after PCV13-implementation, other penicillin non-susceptible serotypes were reported compared to our study; 11A, 15A, 15B/C, 19A, and 35B [32,32]. However, this was in children with acute otitis media. Similar to our results, in Italy serotypes $15 \mathrm{~A}, 19 \mathrm{~A}, 23 \mathrm{~B}$, and $24 \mathrm{~F}$ were reported to be frequently penicillin non-susceptible in IPD isolates [33]. We found nonsusceptibility against erythromycin and tetracycline significantly more frequent in IPD than in carriage strains (Table 4). This is peculiar as erythromycin and tetracycline are not frequently used in children in Belgium. Therefore, this result might be the consequence of antibiotic treatment in adults and consequent transmission of resistant strains to children.

The main limitations of our study are the following. First, the detection of $S$. pneumoniae was performed by culture. Culture is less sensitive than PCR and therefore we might have missed pneumococcal strains that were carried at low density or together with other serotypes. We previously reported PCR-based overall carriage for the first 2 years of carriage monitoring [16,17] and indeed, it was higher $(80.1 \%)$ than culture-based carriage (60.8\%). Therefore, we could have missed changes in individual serotypes and non-susceptibility of strains present at low density. Second, IPD surveillance is performed year-round, whereas carriage monitoring was performed during spring in the first period and winter/spring (five-month period) in the second and third period. Since carriage samples were collected in the period of maximal transmission, we do not expect this to impact our results [34]. Third, age distribution of children with IPD was not exactly the same as in carriage, as no children aged $<6$ months were included in the carriage study. The low number of children younger than 6 months with IPD did not allow to detect or correct for any eventual age-related differences in serotype distribution. As children younger than 6 months old account for only $20 \%$ of all IPD strains and the serotype distribution of IPD strains from children younger than 6 months is comparable to the serotype distribution in children aged 6-30 months (data not shown), we do not expect this to have an impact on our conclusions.

In conclusion, we found that different serotypes dominated in IPD and carriage in Belgian children. The circulating serotypes with highest invasive disease potential (12F, 1, 3, 24B, 24F and 24A) were not frequently carried $(<1.5 \%)$, and only a minority are included in the currently available PCVs. Nevertheless, some of them (serotype $12 \mathrm{~F}, 33 \mathrm{~F}$ ) will be included in the future PCV15 and/or PCV20 [35]. Furthermore, we have found that after a PCV13-to-PCV10 vaccination programme switch, serotype $19 \mathrm{~A}$ increased simultaneously in childhood IPD and carriage, but the antimicrobial nonsusceptibility of this serotype against penicillin was lower in carriage than in IPD. Hence, our results reinforce the need for continued monitoring of carriage and IPD in order to implement the most suitable PCV in the national immunisation programme and in order to optimise the composition of future vaccines.

\section{Declaration of Competing Interest}

The authors declare that they have no known competing financial interests or personal relationships that could have appeared to influence the work reported in this paper.

\section{Appendix A. Supplementary material}

Supplementary data to this article can be found online at https://doi.org/10.1016/j.vaccine.2020.11.044.

\section{References}

[1] Bogaert D, De Groot R, Hermans PW. Streptococcus pneumoniae colonisation: the key to pneumococcal disease. Lancet Infect Dis 2004;4(3):144-54.

[2] Croucher NJ, Lochen A, Bentley SD. Pneumococcal vaccines: host interactions, population dynamics, and design principles. Annu Rev Microbiol 2018;72:521-49.

[3] O'Brien KL et al. Burden of disease caused by Streptococcus pneumoniae in children younger than 5 years: global estimates. Lancet 2009;374 (9693):893-902.

[4] Wahl B et al. Burden of Streptococcus pneumoniae and Haemophilus influenzae type b disease in children in the era of conjugate vaccines: global, regional, and national estimates for 2000-15. Lancet Glob Health 2018;6(7): e744-57.

[5] Geno KA et al. Pneumococcal capsules and their types: Past, present, and future. Clin Microbiol Rev 2015;28(3):871-99.

[6] Tsaban G, Ben-Shimol S. Indirect (herd) protection, following pneumococcal conjugated vaccines introduction: a systematic review of the literature. Vaccine 2017;35(22):2882-91.

[7] Steens A et al. Decreased carriage and genetic shifts in the streptococcus pneumoniae population after changing the seven-valent to the thirteen-valent pneumococcal vaccine in Norway. Pediatr Infect Dis J 2015;34(8):875-83.

[8] Miller E et al. Herd immunity and serotype replacement 4 years after sevenvalent pneumococcal conjugate vaccination in England and Wales: an observational cohort study. Lancet Infect Dis 2011;11(10):760-8.

[9] Mendes da Costa, E., et al., Infectieziekten bij kinderen, die voorkomen kunnen worden door vaccinatie, jaarrapport 2015. 2016, Wetenschappelijk Instituut Volksgezondheid, Brussel.

[10] Robert E, Swennen B. Enquête de couverture vaccinale des enfants de 18 à 24 mois en Fédération Wallonie-Bruxelles (Bruxelles exceptée). 2015, ULB: Bruxelles.

[11] Vandermeulen, C., Hoppenbrouwers, K., Roelants, M., Theeten, H., Braeckman, T., Maertens, K., Blaizot, S., Van Damme, P., Studie van de vaccinatiegraad in Vlaanderen, 2016. 2017, Vlaamse Overheid, Vlaams Agentschap Zorg en Gezondheid.

[12] Hoppenbrouwers, K., et al., Studie van de vaccinatiegraad bij jonge kinderen en adolescenten in Vlaanderen in 2008. 2009, Vlaamse Overheid, Vlaams Agentschap Zorg en Gezondheid.

[13] Robert E, Swennen B. Enquête de couverture vaccinale des enfants de 18 à 24 mois en Communauté Française (Bruxelles excepté) - Novembre 2009. 2010, ULB: Bruxelles.

[14] Desmet S et al. Switch in a childhood pneumococcal vaccination programme from PCV13 to PCV10: a defendable approach?. Lancet Infect Dis 2018;18 (8):830-1.

[15] Wouters I et al. Follow-up of serotype distribution and antimicrobial susceptibility of Streptococcus pneumoniae in child carriage after a PCV13to-PCV10 vaccine switch in Belgium. Vaccine 2019.

[16] Wouters I et al. Nasopharyngeal s. pneumoniae carriage and density in Belgian infants after 9years of pneumococcal conjugate vaccine programme. Vaccine 2018;36(1):15-22.

[17] Wouters I et al. How nasopharyngeal pneumococcal carriage evolved during and after a PCV13-to-PCV10 vaccination programme switch in Belgium, 2016 to 2018. Euro Surveill 2020;25(5).

[18] Desmet $S$ et al. Dynamic changes in Belgian pediatric invasive pneumococcal disease after sequential switch of conjugate vaccine: a national observational study. In press; 2020.

[19] CLSI. CLSI supplement M100. In: Performance Standards for Antimicrobial Susceptibility Testing, P. Wayne, Clinical and Laboratory Standards Institute, Editor. 2017.

[20] EUCAST. Breakpoint tables for interpretation of MICs and zone diameters. Version 7.1, 2017. 2017 [cited 2017; Available from: http://www.eucast. org/fileadmin/src/media/PDFs/EUCAST_files/Breakpoint_tables/ v_7.1_Breakpoint_Tables.pdf.

[21] Lindstrand A et al. Unaltered pneumococcal carriage prevalence due to expansion of non-vaccine types of low invasive potential 8years after vaccine introduction in Stockholm, Sweden. Vaccine 2016:34(38):4565-71.

[22] Brueggemann $A B$ et al. Clonal relationships between invasive and carriage Streptococcus pneumoniae and serotype- and clone-specific differences in invasive disease potential. J Infect Dis 2003:187(9):1424-32.

[23] del Amo E et al. High invasiveness of pneumococcal serotypes included in the new generation of conjugate vaccines. Clin Microbiol Infect 2014;20(7):684-9.

[24] Vissers M et al. Increased carriage of non-vaccine serotypes with low invasive disease potential four years after switching to the 10-valent pneumococcal conjugate vaccine in The Netherlands. PLoS ONE 2018;13(3):e0194823.

[25] Feldman C, Anderson R. Review: current and new generation pneumococcal vaccines. J Infect 2014;69(4):309-25.

[26] Kind \& Gezin. Gebruik van de kinderopvang voor baby's en peuters, formele opvang in. Vlaams Gewest 2017.

[27] ONE. L'One en chiffres - L'accueil Petite Enfance. Couverture de l'accueil. 2017. 
[28] United States Patent Application. Immunogenic compositions comprising conjugated capsular saccharide antigens and uses thereof. 2015 August 2019]; Available from: http://patents.com/us-20150202309.html.

[29] Zemlickova $\mathrm{H}$ et al. Serotype-specific invasive disease potential of Streptococcus pneumoniae in Czech children. J Med Microbiol 2010;59(Pt 9):1079-83.

[30] Balsells E et al. The relative invasive disease potential of Streptococcus pneumoniae among children after PCV introduction: a systematic review and meta-analysis. J Infect 2018;77(5):368-78.

[31] Angoulvant $F$ et al. Trends in antibiotic resistance of Streptococcus pneumoniae and Haemophilus influenzae isolated from nasopharyngeal flora in children with acute otitis media in France before and after 13 valent pneumococcal conjugate vaccine introduction. BMC Infect Dis $2015 ; 15: 236$
[32] Cohen R et al. A 13-year survey of pneumococcal nasopharyngeal carriage in children with acute otitis media following PCV7 and PCV13 implementation. Vaccine 2015;33(39):5118-26.

[33] Camilli, R., et al., Impact of pneumococcal conjugate vaccine (PCV7 and PCV13) on pneumococcal invasive diseases in Italian children and insight into evolution of pneumococcal population structure. Vaccine, 2017. 35(35 Pt B): p. $4587-4593$.

[34] Domenech de Cellès $M$ et al. Unraveling the seasonal epidemiology of pneumococcus. Proc Natl Acad Sci USA 2019;116(5):1802-7.

[35] Lee GM. Pneumococcal Vaccines. Atlanta: CDC; 2019.

[36] Hunter R Paul, Gaston A Michael. Numerical Index of the Discriminatory Ability of Typing Systems: an Application of Simpson's Index of Diversity. J Clin Microbiol 1988;26(10):2465-6. $\quad$ https://doi.org/10.1128/JCM.26.11.24652466.1988. 